OPEN ACCESS

Edited by:

Elisabetta Salvioni

Monzino Cardiology Center (IRCCS), Italy

Reviewed by:

Andrea Segreti,

Campus Bio-Medico University, Italy

Damiano Magri,

Sapienza University of Rome, Italy

${ }^{*}$ Correspondence: Keisuke Miki

miki.keisuke.pu@mail.hosp.go.jp

Specialty section: This article was submitted to

Exercise Physiology, a section of the journal

Frontiers in Physiology

Received: 01 May 2021 Accepted: 18 June 2021

Published: 28 July 2021

Citation:

Miki K, Tsujino K, Maekura R, Matsuki T, Miki M, Hashimoto $H$, Kagawa H, Kawasaki T, Kuge T and Kida $H$ (2021) Oxygen Extraction Based on Inspiratory and Expiratory Gas Analysis Identifies Ventilatory Inefficiency in Chronic Obstructive Pulmonary Disease.

Front. Physiol. 12:703977. doi: 10.3389/fphys.2021.703977

\section{Oxygen Extraction Based on Inspiratory and Expiratory Gas Analysis Identifies Ventilatory Inefficiency in Chronic Obstructive Pulmonary Disease}

\author{
Keisuke Miki ${ }^{1 *}$, Kazuyuki Tsujino ${ }^{1}$, Ryoji Maekura ${ }^{2}$, Takanori Matsuki ${ }^{1}$, Mari Miki ${ }^{1}$, \\ Hisako Hashimoto ${ }^{1}$, Hiroyuki Kagawa ${ }^{1}$, Takahiro Kawasaki ${ }^{1}$, Tomoki Kuge ${ }^{1}$ and \\ Hiroshi Kida ${ }^{1}$ \\ ${ }^{1}$ Department of Respiratory Medicine, National Hospital Organization Osaka Toneyama Medical Center, Toyonaka, Japan, \\ ${ }^{2}$ Graduate School of Health Care Sciences, Jikei Institute, Osaka, Japan
}

Aims: In contrast to cardiovascular disease, low rather than high ventilatory inefficiency, evaluated by the minute ventilation-carbon dioxide output $\left(V^{\prime}{ }^{\prime}-V^{\prime}{ }_{\mathrm{CO} 2}\right)$-slope, has been recognized as being related to greater disease severity in chronic obstructive pulmonary disease (COPD). To better care for patients with cardiopulmonary disease, understanding the physiological correlation between ventilatory inefficiency and exercise limitation is necessary, but remains inadequate. Given that oxygen uptake $\left(V^{\prime} \mathrm{O}_{2}\right)$ evaluated by cardiopulmonary exercise testing (CPET) depends on both the ventilatory capability and oxygen extraction, i.e., the difference between inspiratory and expiratory oxygen concentration $\left(\Delta \mathrm{FO}_{2}\right)$, the aim of this study was to investigate the correlations between $V^{\prime}{ }^{\prime}-V^{\prime} \mathrm{CO}_{2}$-slope and the $\Delta \mathrm{FO}_{2}$ during exercise and their physiological implications in patients with COPD.

Methods: A total of 156 COPD patients (mean age, $70.9 \pm 7.2$ years) with Global Initiative for Chronic Obstructive Lung Disease (GOLD) stages I-IV and 16 controls underwent CPET with blood gas analysis.

Results: With the progression of COPD, mechanical ventilatory constraints together with a slower respiratory frequency led to exertional respiratory acidosis. In GOLD IV cases, (1) decrease in the dependence of reduced peak $V^{\prime}{ }_{\mathrm{O} 2}$ on $V^{\prime} \mathrm{E}$ led to an increase in its dependence on peak $\Delta \mathrm{FO}_{2}$ during exercise; and (2) the $\Delta \mathrm{FO}_{2}-V^{\prime} \mathrm{CO}_{2}$-slope became steeper, correlating with the severity of exertional respiratory acidosis $(r=0.6359, p$ $<0.0001)$. No significant differences in peak exercise $\Delta \mathrm{FO}_{2}$ or $V^{\prime} \mathrm{E}^{-} \mathrm{V}^{\prime} \mathrm{CO}^{-}$-slope were observed among the various GOLD stages. In all subjects, including controls, peak exercise $\Delta \mathrm{FO}_{2}$ had the strongest correlation with the $V^{\prime} \mathrm{E}^{-} V^{\prime} \mathrm{CO}$-slope $(r=-0.8835, p$ $<0.0001$ ) and correlated well with body mass index ( $r=0.3871, p<0.0001)$, although it did not correlate with the heart rate- $V^{\prime} \mathrm{CO}^{-}$-relationship and $V^{\prime} \mathrm{E}$.

Conclusions: Ventilatory efficiency related to $\mathrm{CO}_{2}$ clearance might depend on exertional oxygen extraction in the body. Measuring $\Delta \mathrm{FO}_{2}$ might be a key component for identifying 
ventilatory inefficiency and oxygen availability. Increasing $\Delta \mathrm{FO}_{2}$ would help to improve ventilatory inefficiency and exercise tolerance separately from cardiac and ventilatory capability in COPD patients.

Keywords: acidosis, carbon dioxide, cardiac function, exercise, gas exchange, ventilatory efficiency

\section{BRIEF SUMMARY}

Understanding of the physiological correlation between ventilatory inefficiency and exercise limitation in chronic obstructive pulmonary disease (COPD) patients remains insufficient. The aim of this study was to investigate the correlation between ventilatory inefficiency and exertional variables and their physiological implications in COPD patients, using cardiopulmonary exercise testing and blood gas analysis. With the progression of COPD, the dependence of reduced peak oxygen uptake on the difference between inspiratory and expiratory oxygen concentration $\left(\Delta \mathrm{FO}_{2}\right)$ became relatively high, due to a decrease in its dependence on minute ventilation at peak exercise. Given that both $\mathrm{O}_{2}$ extraction capacity and ventilation affect exercise tolerance, increasing $\Delta \mathrm{FO}_{2}$, as a measure of total oxygen availability in the body, might be the key to improving ventilatory inefficiency related to $\mathrm{CO}_{2}$ clearance, which, in turn, would lead to an increase in exercise tolerance based on the specific dysfunctions in COPD.

\section{INTRODUCTION}

A better ventilatory response to exercise is helpful for improving exercise tolerance and exertional dyspnea in patients with cardiopulmonary diseases. Ventilatory inefficiency, indicated by a higher slope of the minute ventilation $\left(V_{\mathrm{E}}^{\prime}\right)$ vs. volume of exhaled carbon dioxide $\left(V^{\prime} \mathrm{CO}_{2}\right)$ relationship during exercise has been recognized as an index of greater disease severity and worse outcomes in cardiovascular diseases, such as heart failure (HF) and pulmonary arterial hypertension (PAH) (Wasserman et al., 1997; Sue, 2011; Dubé et al., 2016; Weatherald and Laveneziana, 2018; Nayor et al., 2020). In such diseases, excess ventilation is one of the most frequently recognized features to compensate for cardiovascular impairments, with the increase in respiratory frequency $\left(f_{\mathrm{R}}\right)$, implying ventilatory inefficiency. In contrast, chronic obstructive pulmonary disease (COPD), in which cardiovascular impairment is not the primary feature, results in a higher ventilatory demand to compensate for the gas-exchange disorder, especially with the progression of the disease (Neder et al., 2017; Weatherald et al., 2018). Unexpectedly, however, it has been reported that not a high, but a low $V_{\mathrm{E}}^{\prime}-V^{\prime} \mathrm{CO}^{-}$ slope in COPD is associated with increasing COPD severity due to ventilatory abnormalities and mechanical constraints (Neder et al., 2015). To better care for patients with cardiopulmonary

\footnotetext{
Abbreviations: CPET, cardiopulmonary exercise testing; $\triangle \mathrm{FO}_{2}$, difference between inspiratory and expiratory oxygen concentrations; GOLD, Global Initiative for Chronic Obstructive Lung Disease; ICS, inhaled corticosteroid; LABA, long-acting $\beta 2$-agonist; LAMA, long-acting muscarinic antagonist; SABA, short-acting $\beta 2$-agonist; SAMA, short-acting muscarinic antagonist.
}

diseases, a deeper understanding of the physiological implication of the $V_{\mathrm{E}}^{\prime}{ }^{\prime} V^{\prime}{ }_{\mathrm{CO} 2}$ relationship and what is acceptable in common in the different scenarios may be needed.

In cardiopulmonary exercise testing (CPET), only gas flow and the concentrations of oxygen $\left(\mathrm{O}_{2}\right)$ and carbon dioxide $\left(\mathrm{CO}_{2}\right)$ are directly measured, with all other parameters being calculated using these measurements (Wasserman et al., 2012; Laviolette and Laveneziana, 2018). Peak oxygen uptake ( $V^{\prime}{ }^{2}$ 2) obtained from symptom-limited incremental CPET is an excellent indicator of exercise tolerance in cardiopulmonary diseases (Guazzi et al., 2012). Although $V_{\mathrm{O} 2}^{\prime}$ is calculated using the product of $V_{\mathrm{E}}^{\prime}$ and the difference between inspiratory $\mathrm{O}_{2}$ concentration $\left(\mathrm{FiO}_{2}\right)$ and expiratory $\mathrm{O}_{2}$ concentration $\left(\mathrm{FeO}_{2}\right)$ $\left(\Delta \mathrm{FO}_{2}\right)$ (Wasserman et al., 2012; Laviolette and Laveneziana, 2018), little is known about the response of $\Delta \mathrm{FO}_{2}$ to exertion and its relationship with ventilatory inefficiency.

The aim of this study, which included COPD patients across all Global Initiative for Chronic Obstructive Lung Disease (GOLD) stages and control subjects, was 1) to investigate whether the response of $\Delta \mathrm{FO}_{2}$ as a measure of $\mathrm{O}_{2}$ extraction reflects the patterns of cardiopulmonary responses, including an evaluation of respiratory or metabolic acidosis, during incremental CPET together with blood gas analysis, and 2) to clarify the physiological implication of $V_{\mathrm{E}}^{\prime}$ in relation to $\mathrm{CO}_{2}$ clearance, evaluated as the $V_{\mathrm{E}}^{\prime}-V_{\mathrm{CO}}^{\prime}$-slope and the absolute values at the lowest $V_{\mathrm{E}}^{\prime} / V^{\prime}{ }_{\mathrm{CO} 2}$ during exercise, to investigate whether the $V^{\prime}{ }^{-}-V^{\prime}{ }^{\prime}{ }^{2}$ relationship correlates with the $\Delta \mathrm{FO}_{2}$ and clinical or cardiopulmonary variables.

\section{METHODS}

\section{Subjects}

CPETs were performed in 4,557 patients with exertional dyspnea at our institution between May 1997 and August 2020. In this retrospective study, COPD patients and control subjects, who underwent CPET using a treadmill with an exertional evaluation of arterial blood gases and whose full data were stored, were selected. The diagnosis of COPD was confirmed based on the classification of the severity of airflow limitation in 2020 GOLD guidelines ${ }^{1}$. The exclusion criteria were patients who had absolute contraindications to clinical exercise testing (European Respiratory Society, 1997; Radtke et al., 2019), and those who had had a COPD exacerbation within the 2 months before CPET. Patients with comorbidities (e.g., severe cardiovascular disease, malignant tumors, active infection, asthma, pulmonary

\footnotetext{
${ }^{1} 2020$ Global Strategy for Prevention, Diagnosis, and Management of COPD. Global Initiative for Chronic Obstructive Lung Disease. Available online at: https:// goldcopd.org/gold-reports/ (accessed December 20, 2020).
} 
fibrosis, or neuromuscular disease) were also excluded. Agematched control subjects with normal pulmonary function were recruited. No major cardiopulmonary diseases that could affect the results of CPETs were confirmed in the control subjects. This study included data from previous ethically-approved studies performed as screening for studies on COPD or control subjects at our institution. Thus, 156 patients with stable COPD and 16 control subjects were included in the study. All patients and control subjects provided written informed consent to undergo the protocols before the actual CPET. The institutional review board of the National Hospital Organization Osaka Toneyama Medical Center approved the study protocol, including the use of previous data sets (approval number: TNH-R-2020025), and the protocol was in accordance with the Declaration of Helsinki for experiments involving human subjects.

\section{Pulmonary Function Tests}

Post-bronchodilator spirometry (CHESTAC 8800; CHEST M.I. Inc., Tokyo, Japan) was performed before exercise according to the recommendations of the American Thoracic Society (1995).

\section{Cardiopulmonary Exercise Testing (CPET)}

Symptom-limited incremental exercise tests were conducted on a treadmill using the Sheffield protocol or the two modified Sheffield protocols, as previously described (European Respiratory Society, 1997; Miki et al., 2009; Radtke et al., 2019). The exercise protocol that was likely to result in termination of the exercise test in about $8-10$ min was selected based on the patient's daily activities and pulmonary function test results [especially the forced expiratory volume in $1 \mathrm{~s}\left(\mathrm{FEV}_{1}\right)$ ]. Preexercise resting measurements were obtained during the steadystate period after at least $3 \mathrm{~min}$ of breathing through a mask. Exercise tests were performed without encouragement, especially during exercise to obtain reliable data, and were discontinued at subject exhaustion or signs indicating that exercise should stop. Gas exchange measurements were performed with the Aero monitor AE310S (Minato Medical Science Co., Ltd, Osaka, Japan): values of $V_{\mathrm{E}}^{\prime}, V^{\prime}{ }_{\mathrm{O} 2}, V^{\prime}{ }_{\mathrm{CO} 2}, f_{\mathrm{R}}$, tidal volume $\left(V_{\mathrm{T}}\right)$, the ratio of inspiratory time to total respiratory cycle time ( $\mathrm{Ti} / \mathrm{Ttot})$, physiological dead space/tidal volume ratio $\left(V_{\mathrm{D}} / V_{\mathrm{T}}\right), \mathrm{O}_{2}$ pulse [ $V^{\prime}{ }^{\prime} 2$ /heart rate $\left.(\mathrm{HR})\right], \Delta \mathrm{FO}_{2}$, and end-tidal $\mathrm{CO}_{2}$ pressure $\left(\mathrm{PetCO}_{2}\right)$ were measured breath-by-breath and collected as 30$\mathrm{s}$ averages at rest, at $1 \mathrm{~min}$ and at 3-min intervals during exercise, and at the end of the exercise. Dyspnea intensity (10-point modified Borg category-ratio scale) and arterial blood samples for blood gas analyses, and plasma lactate assessments were collected at rest, during the last $15 \mathrm{~s}$ of the $1 \mathrm{~min}$ and at 3-min intervals, and at the end of the exercise, as previously described (Miki et al., 2009). CPETs were performed by three or four operators, and the data were analyzed by all the respiratory medicine house staff once a week. The $V^{\prime}{ }^{\prime}-V^{\prime} \mathrm{CO}_{2}$-slope was calculated by linear regression, excluding the non-linear part of the data after the onset of the respiratory compensation point (Figure 1). When no respiratory compensation could be identified, the $V_{\mathrm{E}}^{\prime}-V_{\mathrm{CO} 2}^{\prime}$-slope was calculated from the data recorded from the start to the end of the exercise. Similarly, the bicarbonate ion $\left(\mathrm{HCO}_{3}^{-}\right)-V^{\prime} \mathrm{CO}_{2}$-slope, Ti/Ttot- $V^{\prime} \mathrm{CO}_{2}$-slope,
$\mathrm{HR}-V^{\prime}{ }_{\mathrm{CO} 2}$-slope, and $\mathrm{PaCO}_{2}-V^{\prime} \mathrm{CO}_{2}$-slope were calculated; that is, the linear phase of each parameter- $V^{\prime}{ }_{\mathrm{CO} 2}$ relationship was determined from each parameter $-V^{\prime}{ }^{\prime}{ } 2$ plot (each parameter during exercise was plotted on the Y-axis and the $V^{\prime}{ }^{\prime}{ }_{2}$ was plotted on the $\mathrm{X}$-axis) (Figure 1). Positive and negative $\mathrm{HCO}_{3}^{-}$$V^{\prime} \mathrm{CO}_{2}$-slopes were defined as exertional respiratory acidosis and exertional metabolic acidosis patterns, respectively. The $V_{\mathrm{E}^{-}}^{\prime}$ $V^{\prime} \mathrm{CO}_{2}$-intercept was defined as the nonzero point on the Yaxis, that is, $V_{\mathrm{E}}^{\prime}$ (Figure 1). The $V_{\mathrm{E}}^{\prime} / V^{\prime} \mathrm{CO}_{2}$-nadir was defined as the lowest value during exercise. Positive and negative Ti/Ttot$V^{\prime} \mathrm{CO} 2$-slopes were defined as non-prolonged expiration and prolonged expiration patterns, respectively. Predicted maximal voluntary ventilation $(\mathrm{MVV})$ was calculated as $\mathrm{FEV}_{1} \times 35$. Predicted maximum HR was calculated as 220-age in years (Wasserman et al., 2012).

\section{Statistical Analysis}

Variables are expressed as means \pm standard deviation (SD) unless otherwise stated. For continuous variables, (1) the Wilcoxon rank-sum test was used for comparisons between controls and patients of all GOLD stages, (2) the Kruskal-Wallis test was used for comparison among the groups consisting of the four GOLD stages and controls, followed by the Steel-Dwass test to carry out between-group comparisons, and (3) univariate analysis using Spearman's rank correlation coefficient was used to study the correlations between clinical variables. R squared was used to confirm how close the data were to the fitted regression line. The chi-squared test was used for categorical variables. A $p<0.05$ was considered significant. Statistical analyses were performed using JMP software, version 11 (SAS Institute Inc., Cary, NC, USA).

\section{RESULTS}

A total of 156 patients distributed across all GOLD stages and 16 controls constituted the entire study sample (Table 1). All patients and controls were well-matched for age, sex, and body mass index (BMI), although BMI was lower in GOLD III and IV than in GOLD I groups.

\section{Pathophysiological Response and $\mathrm{O}_{2}$ Extraction During Exercise}

Incremental exercise parameters at peak exercise in COPD patients and controls are shown in Table 2. From controls to GOLD IV, (1) the ratio of the prolonged expiration pattern ( $p$ $<0.0001)$ and the ratio of the respiratory acidosis pattern $(p$ $=0.0025)$ increased during exercise, and (2) a slower $f_{\mathrm{R}}(p$ $=0.0004)$ and lower $V_{\mathrm{T}}(p<0.0001)$, i.e., slow and shallow breathing, were confirmed, along with the lower Ti/Ttot, i.e., prolonged expiration $(p<0.0001)$, all of which led to the increased $\mathrm{HCO}_{3}^{-}$level $(p<0.0001)$ with the higher arterial carbon dioxide tension $\left(\mathrm{PaCO}_{2}\right)$ level $(p<0.0001)$ and the lower plasma lactate level $(p<0.0001)$, i.e., respiratory acidosis (Table 2). In GOLD IV, (1) although no significant differences in $\mathrm{pH}$ levels among GOLD stages were seen in this study population, $\mathrm{PaCO}_{2}$ was higher, and plasma lactate level was lower than in the other groups (Table 2); and (2) the peak $V^{\prime}{ }^{\prime} 2$ was 

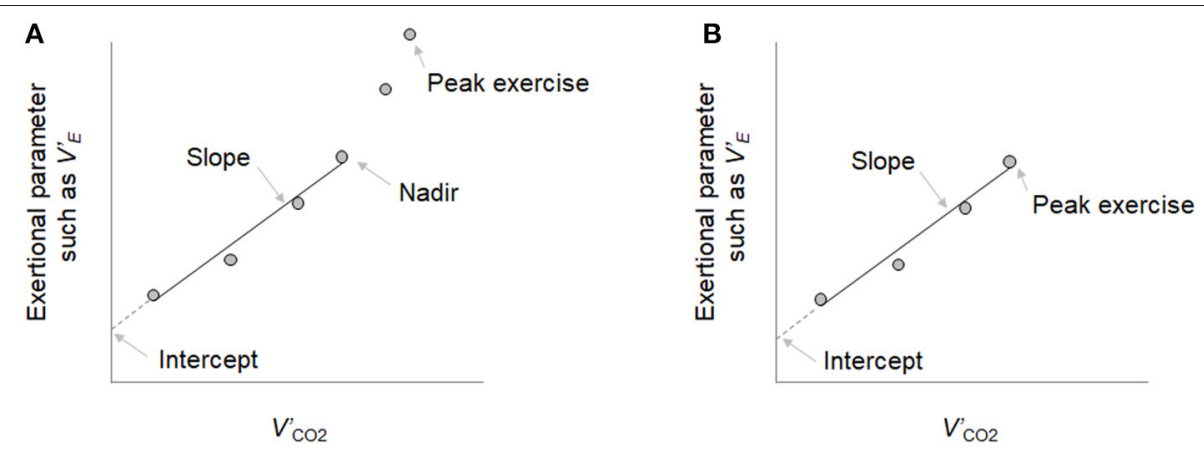

FIGURE 1 | The exertional parameters-carbon dioxide output $\left(V^{\prime} \mathrm{CO}_{2}\right)$ relationship. (A) When the respiratory compensation point (RCP) was observed during cardiopulmonary exercise testing, the minute ventilation $\left(V^{\prime} \mathrm{E}\right)-V^{\prime} \mathrm{CO}_{2}$-slope was calculated by linear regression, excluding the nonlinear part of the data after the onset of the RCP. (B) When no RCP could be identified, the $V^{\prime} \mathrm{E}^{-} V^{\prime} \mathrm{CO}^{-}$-slope was calculated from the data recorded from the start of exercise to the end of exercise. Similarly, the bicarbonate ion $\left(\mathrm{HCO}_{3}^{-}\right)-V^{\prime} \mathrm{CO}_{2}$-slope, the ratio of inspiratory time to total respiratory cycle time ( $T \mathrm{i} / T$ tot)- $V^{\prime} \mathrm{CO} 2$-slope, heart rate ( $\left.\mathrm{HR}\right)-V^{\prime} \mathrm{CO} 2$-slope, and $\mathrm{PaCO}_{2}-V^{\prime} \mathrm{CO}_{2}$-slope were determined in each parameter $-V^{\prime} \mathrm{CO} 2$ plot. This is an original figure. No permission is required.

TABLE 1 | Baseline characteristics and resting pulmonary function of controls and COPD patients classified by the Global Initiative for Chronic Obstructive Lung Disease (GOLD).

\begin{tabular}{|c|c|c|c|c|c|c|}
\hline \multirow[t]{2}{*}{ Subject $(n)$} & \multirow[t]{2}{*}{ Controls (16) } & \multirow[t]{2}{*}{ All COPD patients (156) } & \multicolumn{4}{|c|}{ GOLD spirometric severity } \\
\hline & & & I (11) & II (37) & III (66) & IV (42) \\
\hline Age, y & $68.8(10.3)$ & $70.9(7.2)$ & $69.5(6.2)$ & $72.1(8.1)$ & $72.6(6.6)$ & $67.7(6.5)^{t_{c}}$ \\
\hline Sex, male/female $(n)$ & $13 / 3$ & $142 / 14$ & $11 / 0$ & $33 / 4$ & $58 / 8$ & $40 / 2$ \\
\hline $\mathrm{BMl}, \mathrm{kg} \cdot \mathrm{m}^{-2}$ & $21.3(2.9)$ & $20.9(3.3)$ & $23.7(1.7)$ & $21.6(3.2)$ & $20.4(3.6)^{t_{a}}$ & $20.3(3.0)^{\dagger_{a}}$ \\
\hline \multicolumn{7}{|l|}{ Pulmonary function test } \\
\hline $\mathrm{FEV}_{1}, \mathrm{~L}$ & $2.48(0.73)$ & $1.13(0.54)^{\star}$ & $2.48(0.34)$ & $1.55(0.30)^{\star}{ }^{\dagger a}$ & $0.97(0.22)^{\star} t_{a}+b$ & $0.67(0.12)^{*} t_{a} t_{b} t_{c}$ \\
\hline$\% \mathrm{FEV}_{1}, \%$ predicted & $95.9(19.2)$ & $44.1(19.4)^{\star}$ & $91.6(6.2)$ & $61.2(7.6)^{\star}+a$ & $38.7(5.7)^{\star}+a+b$ & $24.9(3.8)^{\star} t_{a} t_{b} t_{c}$ \\
\hline $\mathrm{FEV}_{1} / \mathrm{FVC}, \%$ & $80.5(8.7)$ & $45.1(12.5)^{\star}$ & $62.0(4.7)^{\star}$ & $54.3(10.0)^{\star}$ & $43.1(8.4)^{\star} t_{a} t_{b}$ & $34.7(9.0)^{\star} t_{a} t_{b} t_{c}$ \\
\hline VC, L & $3.34(0.99)$ & $2.87(0.78)^{\star}$ & $4.24(0.75)$ & $3.12(0.68)^{\dagger_{a}}$ & $2.71(0.66)^{t_{a} t_{b}}$ & $2.53(0.59)^{\star} t_{a} t_{b}$ \\
\hline$\%$ VC, \% & $107.2(22.6)$ & $91.5(20.6)^{\star}$ & $128.7(15.6)$ & $101.0(15.9)^{\dagger a}$ & $88.6(16.2)^{\star} t_{a} t_{b}$ & $78.0(16.1)^{\star} t_{a} t_{b} t_{c}$ \\
\hline IC, L & $2.28(0.84)$ & $1.78(0.61)^{\star}$ & $2.77(0.74)$ & $2.05(0.43)^{\dagger a}$ & $1.64(0.56)^{\star}+a+b$ & $1.51(0.41)^{\star}+a+b$ \\
\hline \multicolumn{7}{|l|}{ Medications $(n)^{\ddagger}$} \\
\hline LAMA/LABA/ICS/SAMA/SABA/Theo & - & 39/28/33/41/11/63 & $1 / 1 / 0 / 0 / 0 / 1$ & $11 / 6 / 8 / 5 / 3 / 12$ & $19 / 15 / 15 / 20 / 3 / 27$ & $8 / 6 / 10 / 16 / 5 / 23$ \\
\hline \multicolumn{7}{|l|}{ Primary comorbidities ( $n$ ) } \\
\hline Old myocardial infarction & - & 5 & 1 & 0 & 2 & 2 \\
\hline Previous angina pectoris & - & 9 & 1 & 1 & 4 & 3 \\
\hline Persistent atrial fibrillation & - & 3 & 0 & 1 & 2 & 0 \\
\hline Lower extremity artery disease & - & 3 & 0 & 1 & 1 & 1 \\
\hline Past tuberculosis disease & - & 3 & 0 & 0 & 2 & 1 \\
\hline
\end{tabular}

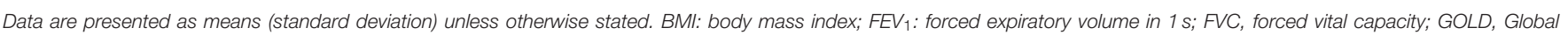

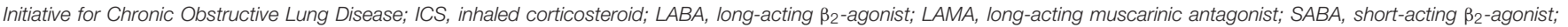

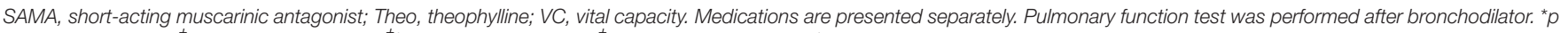
$<0.05$ vs. controls; ${ }^{\dagger a} p<0.05$ vs. GOLD I; ${ }^{t b} p<0.05$ vs. GOLD II; ${ }^{+c} p<0.05$ vs. GOLD III. ${ }^{\ddagger}$ Medication data were not obtained in four patients.

lower than in the other groups (Table 2) and dependence of the reduced peak $V^{\prime}{ }^{\prime} 2$ on $\Delta \mathrm{FO}_{2}$ became relatively high because of its decreased dependence on $V_{\mathrm{E}}^{\prime}$ (Table 3 ). In addition, the ratio of $\Delta \mathrm{FO}_{2}$ to $V_{\mathrm{E}}^{\prime}$ at peak exercise was higher in the GOLD IV group than in the other groups (Table 2). Although there were no significant intrastage differences in peak exercise $\Delta \mathrm{FO}_{2}$ and no differences between peak exercise $\Delta \mathrm{FO}_{2}$ and resting $\Delta \mathrm{FO}_{2}$ values among the GOLD stages, the $\Delta \mathrm{FO}_{2}-V^{\prime} \mathrm{CO}_{2}$-slope became steeper with the progression of the COPD stage (Table 2).
The $\Delta \mathrm{FO}_{2}-V^{\prime} \mathrm{CO}_{2}$-slope correlated well with the $\mathrm{PaCO}_{2}-V^{\prime} \mathrm{CO}_{2}$ slope, indicating exertional respiratory acidosis rather than the $\mathrm{PaO}_{2}-V^{\prime} \mathrm{CO}_{2}$-slope, reflecting exertional hypoxemia (Table 4).

\section{Correlations Between Ventilatory Inefficiency and the Assessed Parameters}

Respiratory compensation points during CPET were observed in $9 / 16(56 \%)$ controls, 6/11 (55\%) GOLD I cases, 11/37 (30\%) GOLD II cases, 11/66 (17\%) GOLD III cases, and 9/42 (21\%) 
TABLE 2 | Incremental exercise parameter of controls and COPD patients classified by the Global Initiative for Chronic Obstructive Lung Disease (GOLD).

\begin{tabular}{|c|c|c|c|c|c|c|}
\hline \multirow[b]{2}{*}{ Subject $(n)$} & \multirow[b]{2}{*}{ Controls (16) } & \multicolumn{4}{|c|}{ GOLD spirometric severity } & \multirow[b]{2}{*}{$p$-value } \\
\hline & & I (11) & II (37) & III (66) & IV (42) & \\
\hline \multicolumn{7}{|l|}{ At peak exercise } \\
\hline Dyspnea, Borg scale & $7.1(1.6)$ & $5.8(3.0)$ & $7.1(2.2)$ & $6.8(2.4)$ & $6.8(2.4)$ & 0.7954 \\
\hline$V_{\mathrm{O} 2}^{\prime}, \mathrm{mL} \cdot \mathrm{min}^{-1} \cdot \mathrm{kg}^{-1}$ & $28.1(4.6)$ & $23.6(4.2)$ & $20.0(4.8)^{\star}$ & $16.8(4.7)^{\star}+a+b$ & $12.9(3.4)^{\star} t_{a} t_{b} t_{c}$ & $<0.0001$ \\
\hline$R$ & $1.12(0.10)$ & $1.08(0.10)$ & $1.03(0.09)^{\star}$ & $1.01(0.10)^{*}$ & $0.93(0.09)^{\star} t_{a} t_{b} t_{c}$ & $<0.0001$ \\
\hline$V^{\prime} \mathrm{E}, \mathrm{L} \cdot \mathrm{min}^{-1}$ & $56.6(12.3)$ & $65.9(18.4)$ & $46.8(12.3)^{\dagger a}$ & $36.1(9.1)^{\star}+a+b$ & $26.5(5.6)^{\star}{ }^{+a t_{b} t_{c}}$ & $<0.0001$ \\
\hline$V_{\mathrm{T}}, \mathrm{mL}$ & $1,628(485)$ & $1,925(412)$ & $1,378(303)^{\dagger_{a}}$ & $1,125(309)^{\star} t_{a} t_{b}$ & $944(214)^{\star} t_{a} t_{b} t_{c}$ & $<0.0001$ \\
\hline$f_{\mathrm{R}}$, breaths $\cdot \min ^{-1}$ & $37(7)$ & $35(5)$ & $35(6)$ & $33(6)$ & $29(6)^{\star} t_{b} t_{c}$ & 0.0004 \\
\hline $\mathrm{Ti} / \mathrm{Ttot}$ & $0.49(0.03)$ & $0.46(0.03)$ & $0.42(0.04)^{\star}$ & $0.38(0.04)^{\star}+a t_{b}$ & $0.35(0.04)^{\star} t_{a} t_{b} t_{c}$ & $<0.0001$ \\
\hline$V_{\mathrm{E}}^{\prime} / V^{\prime} \mathrm{O} 2$ & $36.1(3.8)$ & $43.1(7.5)^{\star}$ & $42.4(8.6)$ & $42.7(10.3)^{\star}$ & $39.8(8.0)$ & 0.0281 \\
\hline$V_{E}^{\prime} V^{\prime} V^{\prime} \mathrm{CO}$ & $32.5(4.8)$ & $40.0(6.7)$ & $41.6(9.9)^{*}$ & $42.3(9.5)^{\star}$ & $42.9(9.3)^{\star}$ & 0.0005 \\
\hline$V_{\mathrm{D}} / V_{\mathrm{T}}$ & $0.21(0.05)$ & $0.22(0.04)$ & $0.27(0.07)^{\star}$ & $0.31(0.07)^{\star}+a+b$ & $0.35(0.06)^{\star} t_{a}+b t_{c}$ & $<0.0001$ \\
\hline $1-V^{\prime}{ }^{\prime} / M W, \%$ & $30.6(13.5)$ & $25.4(11.8)$ & $13.8(16.4) *$ & $-7.4(19.9)^{\star}+a t_{b}$ & $-15.4(22.9)^{\star}+a+b$ & $<0.0001$ \\
\hline $\mathrm{HR}$, beats $\cdot \mathrm{min}^{-1}$ & $143(14)$ & $137(17)$ & $132(21)$ & $128(14)^{\star}$ & $120(14)^{\star} t_{a} t_{c}$ & $<0.0001$ \\
\hline HR/predicted maximum HR, \% & $95.2(8.3)$ & $91.3(11.1)$ & $88.5(13.8)$ & $85.7(9.3)^{\star}$ & $79.5(9.3)^{\star} t_{a} t_{b} t_{c}$ & $<0.0001$ \\
\hline $\mathrm{O}_{2}$ pulse, $\mathrm{mL} \cdot$ beats $^{-1}$ & $11.0(2.0)$ & $11.4(3.2)$ & $8.7(2.7)^{\star}$ & $6.9(2.1)^{\star}+a+b$ & $5.7(1.3)^{\star} t_{a} t_{b} t_{c}$ & $<0.0001$ \\
\hline$\Delta \mathrm{FO}_{2,} \%$ & $3.570 .38)$ & $2.96(0.56)^{\star}$ & $2.96(0.58)^{\star}$ & $2.95(0.61)^{\star}$ & $3.08(0.60)^{\star}$ & 0.0057 \\
\hline$\Delta \mathrm{FO}_{2} / V^{\prime} \mathrm{E}, \% \cdot \mathrm{L}^{-1} \cdot \min$ & $0.066(0.015)$ & $0.049(0.020)$ & $0.068(0.024)$ & $0.088(0.033)^{\dagger a}+b$ & $0.122(0.037)^{\star}+a t_{b} t_{c}$ & $<0.0001$ \\
\hline $\mathrm{pH}$ & $7.359(0.031)$ & $7.349(0.032)$ & $7.362(0.038)$ & $7.355(0.039)$ & $7.350(0.033)$ & 0.7082 \\
\hline $\mathrm{PaO}_{2}, \mathrm{mmHg}$ & $82.8(8.7)$ & $65.3(12.0)^{*}$ & $68.3(14.1)^{*}$ & $61.8(11.8)^{\star}$ & $56.1(9.6)^{\star}+b$ & $<0.0001$ \\
\hline $\mathrm{PaCO}_{2}, \mathrm{mmHg}$ & $38.6(3.3)$ & $36.1(3.9)$ & $38.3(5.7)$ & $42.3(6.5)^{t_{a}+b}$ & $46.1(5.8)^{\star} \dagger_{a} t_{b} t_{c}$ & $<0.0001$ \\
\hline $\mathrm{PetCO}_{2}, \mathrm{mmHg}$ & $40.1(5.6)$ & $33.3(5.5)$ & $34.0(6.3)^{\star}$ & $35.9(6.1)$ & $38.3(6.2)^{\dagger b}$ & 0.0022 \\
\hline $\mathrm{HCO}_{3}^{-}, \mathrm{mEq} \cdot \mathrm{L}^{-1}$ & $21.5(2.0)$ & $19.8(1.6)$ & $21.4(2.7)$ & $23.2(2.7)^{\dagger_{a} t_{b}}$ & $25.2(2.8)^{\star} t_{a} t_{b} t_{c}$ & $<0.0001$ \\
\hline Plasma Lactate, $\mathrm{mg} \cdot \mathrm{dL}^{-1}$ & $41.0(14.9)$ & $43.7(17.1)$ & $35.1(15.6)$ & $28.7(11.2)^{\star}+a$ & $22.1(9.0)^{\star} t_{a} t_{b} t_{c}$ & $<0.0001$ \\
\hline \multicolumn{7}{|l|}{ During exercise } \\
\hline Expiration pattern, prolonged/non-prolonged/ (n) & $1 / 15$ & $0 / 11$ & $7 / 30$ & $30 / 36$ & $32 / 10$ & $<0.0001$ \\
\hline Exertional acidosis pattern, respiratory/metabolic $(n)$ & $1 / 15$ & $1 / 10$ & $7 / 32$ & $21 / 45$ & $19 / 23$ & 0.0025 \\
\hline$V^{\prime}{ }^{\prime}-V^{\prime} \mathrm{CO}_{2}$-slope & $28.0(4.9)$ & $35.2(6.1)^{\star}$ & $35.6(8.9)^{\star}$ & $35.5(11.3)^{\star}$ & $33.6(10.9)$ & 0.0081 \\
\hline$V^{\prime}$ / $V^{\prime} \mathrm{CO}_{2}$-nadir & $32.2(4.9)$ & $38.7(6.0)^{\star}$ & $41.4(9.8)^{\star}$ & $42.2(9.4)^{*}$ & $42.4(9.4)^{\star}$ & 0.0003 \\
\hline$V^{\prime}{ }^{\prime}-V^{\prime} \mathrm{CO}_{2}$-intercept, $\mathrm{L} \cdot \mathrm{min}^{-1}$ & $6.7(2.4)$ & $5.8(2.3)$ & $6.4(2.7)$ & $6.2(3.1)$ & $6.2(2.5)$ & 0.9772 \\
\hline$V_{E}^{\prime} / V^{\prime} \mathrm{CO}_{2}-$ rest minus nadir & $27.0(14.1)$ & $20.9(8.4)$ & $20.3(9.6)$ & $20.3(9.0)$ & $19.6(10.6)$ & 0.2784 \\
\hline$\Delta \mathrm{FO}_{2}, \%$ peak exercise minus rest & $1.29(0.62)$ & $0.72(0.52)$ & $0.84(0.52)$ & $0.88(0.50)$ & $0.98(0.52)$ & 0.0505 \\
\hline$\Delta \mathrm{FO}_{2}-V^{\prime} \mathrm{CO}_{2}$-slope, $\% \cdot L^{-1} \cdot \min$ & $0.91(0.39)$ & $0.53(0.47)$ & $0.91(0.50)$ & $1.42(1.05)^{t_{a}+b}$ & $2.46(0.19)^{\star}+a t_{b} t_{c}$ & $<0.0001$ \\
\hline HR-V'co2-slope, beats $\cdot L^{-1}$ & $41.6(9.0)$ & $38.6(12.8)$ & $47.7(22.5)$ & $65.5(31.6)^{\star} t_{a} t_{b}$ & $77.6(37.0)^{*}+a+b$ & $<0.0001$ \\
\hline
\end{tabular}

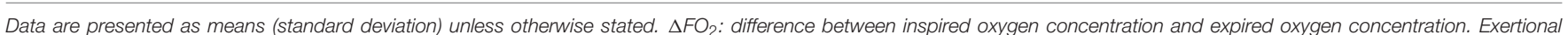

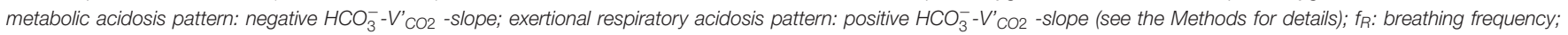

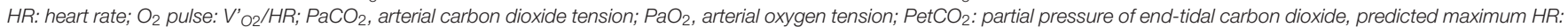

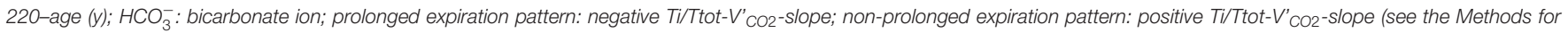

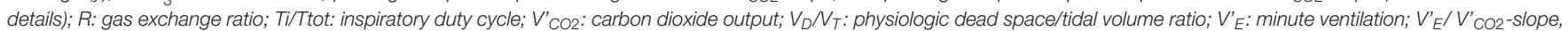

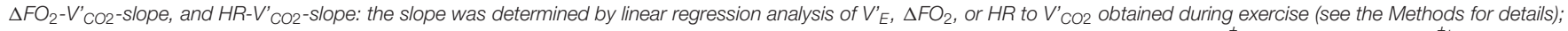

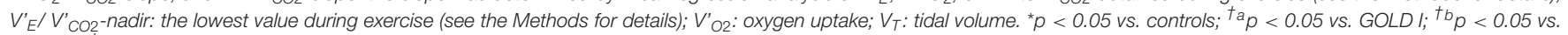
GOLD II; ${ }^{+c} p<0.05$ vs. GOLD III. Estimated maximal voluntary ventilation (MVV) (L.min $\left.{ }^{-1}\right)$ was equal to forced expiratory volume in $1 \mathrm{~s}($ (FEV 1$) \times 35$.

TABLE 3 | Dominant correlations of the peak oxygen uptake classified by the Global Initiative for Chronic Obstructive Lung Disease (GOLD).

\begin{tabular}{|c|c|c|c|c|c|c|c|c|c|c|}
\hline \multirow{3}{*}{ Subject $(n)$} & & & \multicolumn{8}{|c|}{ GOLD spirometric severity } \\
\hline & \multicolumn{2}{|c|}{ Controls (16) } & \multicolumn{2}{|c|}{ I (11) } & \multicolumn{2}{|c|}{ II (37) } & \multicolumn{2}{|c|}{ III (66) } & \multicolumn{2}{|c|}{ IV (42) } \\
\hline & $r^{2}$ & $p$-value & $r^{2}$ & $p$-value & $r^{2}$ & $p$-value & $r^{2}$ & $p$-value & $r^{2}$ & $p$-value \\
\hline$V_{E}^{\prime}$, at peak exercise, $L \cdot \min ^{-1}$ & 0.62 & 0.0003 & 0.56 & 0.0082 & 0.52 & $<0.0001$ & 0.57 & $<0.0001$ & 0.39 & $<0.0001$ \\
\hline$\Delta \mathrm{FO}_{2}$, at peak exercise, $\%$ & 0.15 & 0.1556 & 0.28 & 0.0947 & 0.35 & 0.0001 & 0.34 & $<0.0001$ & 0.29 & $<0.0001$ \\
\hline
\end{tabular}

$\Delta \mathrm{FO}_{2}$, difference between inspired oxygen concentration and expired oxygen concentration; $V_{E}^{\prime}$, minute ventilation. 
TABLE 4 | Correlations of $\triangle \mathrm{FO}_{2}-V^{\prime} \mathrm{CO}_{2}$-slope classified by the Global Initiative for Chronic Obstructive Lung Disease (GOLD).

\begin{tabular}{|c|c|c|c|c|c|c|c|c|c|c|}
\hline \multirow{3}{*}{ Subject $(n)$} & & & \multicolumn{8}{|c|}{ GOLD spirometric severity } \\
\hline & \multicolumn{2}{|c|}{ Controls (16) } & \multicolumn{2}{|c|}{ I (11) } & \multicolumn{2}{|c|}{ II (37) } & \multicolumn{2}{|c|}{ III (66) } & \multicolumn{2}{|c|}{ IV (42) } \\
\hline & $r$ & $p$-value & $r$ & $p$-value & $r$ & $p$-value & $r$ & $p$-value & $r$ & $p$-value \\
\hline $\mathrm{PaO}_{2}-V^{\prime} \mathrm{CO}_{2}$-slope, $\mathrm{mmHg} \cdot \mathrm{L}^{-1} \cdot \min$ & -0.1480 & 0.5987 & 0.3158 & 0.3441 & 0.0244 & 0.8861 & -0.1617 & 0.1946 & -0.2137 & 0.1742 \\
\hline $\mathrm{PaCO}_{2}-V^{\prime} \mathrm{CO}_{2}$-slope, $\mathrm{mmHg} \cdot \mathrm{L}^{-1} \cdot \min$ & 0.3220 & 0.2419 & 0.3586 & 0.2788 & 0.1903 & 0.2593 & 0.4646 & $<0.0001$ & 0.6359 & $<0.0001$ \\
\hline Lactate-V'co2-slope, $\mathrm{mg} \cdot \mathrm{dL}^{-1} \cdot \mathrm{L}^{-1} \cdot \min$ & -0.0448 & 0.8740 & -0.5904 & 0.0558 & -0.1745 & 0.3015 & -0.2724 & 0.0269 & -0.0318 & 0.8418 \\
\hline $\mathrm{pH}-V^{\prime} \mathrm{CO}_{2}$-slope, $\mathrm{mmHg} \cdot \mathrm{L}^{-1} \cdot \min$ & -0.1289 & 0.6471 & 0.2171 & 0.5214 & -0.1407 & 0.4061 & -0.2482 & 0.0445 & -0.3470 & 0.0243 \\
\hline$f_{\mathrm{R}}-V^{\prime} \mathrm{CO} 2^{-}$-slope, breaths $\cdot \mathrm{L}^{-1}$ & -0.1505 & 0.5923 & -0.3945 & 0.2299 & -0.2223 & 0.1860 & 0.0324 & 0.7961 & -0.3524 & 0.0221 \\
\hline
\end{tabular}

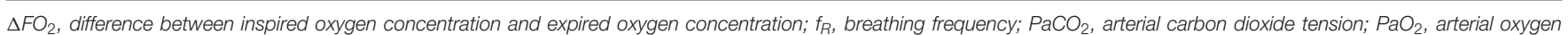

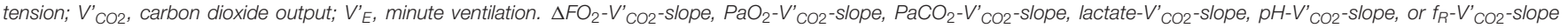
were determined by linear regression analysis of $\Delta \mathrm{FO}_{2}, \mathrm{PaO}_{2}, \mathrm{PaCO}_{2}$, plasma lactate level, $\mathrm{pH}$, or $\mathrm{f}_{\mathrm{R}}$ to $\mathrm{V}^{\prime} \mathrm{CO} 2$ obtained during exercise (see the Methods for details).

GOLD IV cases. No significant intrastage differences were confirmed in $V_{\mathrm{E}}^{\prime} / V_{\mathrm{CO} 2}^{\prime}$ at peak exercise, $V_{\mathrm{E}}^{\prime}-V^{\prime} \mathrm{CO}_{2}$-slope, the $V_{\mathrm{E}}^{\prime} / V_{\mathrm{CO} 2}^{\prime}$-nadir, $V_{\mathrm{E}}^{\prime}-V^{\prime} \mathrm{CO}^{\prime}$-intercept, and the difference between the $V_{\mathrm{E}}^{\prime} / V^{\prime}{ }_{\mathrm{CO} 2}$-nadir and $V_{\mathrm{E}}^{\prime} / V^{\prime}{ }_{\mathrm{CO} 2}$ at rest among the four GOLD stages, although the $V_{\mathrm{D}} / V_{\mathrm{T}}$ at peak exercise was higher in GOLD stage III and IV patients than at the milder stages and in controls (Table 2). As shown in Figures 2A-D, in both COPD patients and controls, the $V_{\mathrm{E}}^{\prime}-V^{\prime} \mathrm{CO}^{-}$-slope correlated strongly with the peak exercise $\Delta \mathrm{FO}_{2}$ (oxygen extraction), compared with peak $V^{\prime}{ }_{\mathrm{O} 2}$ (exercise tolerance), $V_{\mathrm{E}}^{\prime}$ (ventilatory capability), and $\mathrm{O}_{2}$-pulse (cardiac function). Correlations of the $V_{\mathrm{E}}^{\prime}-V^{\prime}{ }_{\mathrm{CO}}$-slope with the variables evaluated in the present study in all the subjects, including controls (Table 5 and Figures 2A-D), indicated no correlation with dyspnea level at peak exercise $(\mathrm{r}=0.0934)$, a weak correlation with peak $V_{\mathrm{O} 2}^{\prime}(\mathrm{r}=$ -0.3219 ), slight correlation with $f_{\mathrm{R}}$ at peak exercise (which was the only ventilatory parameter) $(r=0.2122)$, weak correlation with $\mathrm{O}_{2}$-pulse at peak exercise as a cardiovascular parameter $(\mathrm{r}=-0.3331)$, good correlations with $\mathrm{PaCO}_{2}(\mathrm{r}=-0.5250)$, and $\mathrm{PetCO}_{2}(\mathrm{r}=-0.8542)$ as gas exchange parameters related to $\mathrm{CO}_{2}$ clearance, the strongest correlation with $\Delta \mathrm{FO}_{2}(\mathrm{r}=$ -0.8835 ) at peak exercise as a gas exchange parameter related to $\mathrm{O}_{2}$ extraction, and weak correlation with BMI $(\mathrm{r}=-0.2906)$. The correlations of the $V_{\mathrm{E}}^{\prime} / V^{\prime} \mathrm{CO}_{2}$-nadir in all subjects were almost the same as those of the $V^{\prime}{ }^{\prime}-V^{\prime} \mathrm{CO}_{2}$-slope (Table 5). Only slight correlation was observed between the $V_{\mathrm{E}}^{\prime} / V^{\prime} \mathrm{CO}_{2}$-intercept and the peak exercise $\Delta \mathrm{FO}_{2}$ in all subjects including controls $(\mathrm{r}$ $=0.2155)$.

\section{Correlations Between $\mathrm{O}_{2}$ Extraction and the Various Parameters}

In all the study subjects including controls and in only COPD patients (Table 6), peak exercise $\Delta \mathrm{FO}_{2}$ (1) correlated well with the gas exchange parameters related to $\mathrm{CO}_{2}$ clearance such as $V_{\mathrm{E}}^{\prime} / V_{\mathrm{CO} 2}^{\prime}, V_{\mathrm{D}} / V_{\mathrm{T}}, \mathrm{PaCO}_{2}$, and $\mathrm{PetCO}_{2}$ at peak exercise; (2) did not correlate with $V_{\mathrm{E}}^{\prime}$ at peak exercise; (3) correlated more significantly with $\mathrm{O}_{2}$-pulse at peak exercise than with peak $V^{\prime}{ }_{\mathrm{O} 2}$ or HR- $V^{\prime}$ CO2-slope; and (4) correlated positively with BMI. However, there was no correlation between $\mathrm{BMI}$ and peak $V^{\prime}{ }_{\mathrm{O} 2}$
( $\mathrm{r}=0.0896, p=0.2425$ for all subjects; $\mathrm{r}=0.0981 p=0.2232$ for COPD patients).

\section{DISCUSSION}

All subjects across all four GOLD COPD stages and controls underwent CPET with an evaluation of exertional acidosis to investigate the cardiopulmonary response of $\Delta \mathrm{FO}_{2}$ to exercise and the physiological implications of the $V_{\mathrm{E}}^{\prime}-V^{\prime}{ }_{\mathrm{CO} 2}$ relationship. The main findings of this study were as follows. First, as COPD progressed, (1) patients with more severe COPD had a slow and shallow respiratory pattern, because of the mechanical constraints on $V_{\mathrm{T}}$, which led to exertional respiratory acidosis, and greater dependence of their reduced peak $V^{\prime}{ }^{\prime} 2$ on peak exercise $\Delta \mathrm{FO}_{2}$ due to the decreased dependence on $V_{\mathrm{E}}^{\prime}$. In addition, the ratio of $\Delta \mathrm{FO}_{2}$ to $V_{\mathrm{E}}^{\prime}$ was higher with more severe COPD; and 2) although there was no significant difference in peak exercise $\Delta \mathrm{FO}_{2}$ among the GOLD stages, the $\Delta \mathrm{FO}_{2}-V^{\prime} \mathrm{CO}^{-}$ slope became steeper and correlated well with the slope of the exertional respiratory acidosis level. Second, peak exercise $\Delta \mathrm{FO}_{2}$ as a gas exchange parameter related to $\mathrm{O}_{2}$ extraction had the strongest correlation with ventilatory inefficiency related to $\mathrm{CO}_{2}$ clearance among the gas exchange parameters, mechanical ventilatory parameters, exercise tolerance and exertional cardiac function in all subjects including controls. Third, peak exercise $\Delta \mathrm{FO}_{2}$ did not contribute significantly to $V_{\mathrm{E}}^{\prime}$.

The excess or reduced ventilatory response to exercise based on the ventilatory capability in each cardiopulmonary disease might result from compensation for the pathophysiological condition that each impairment causes during exercise. In patients with $\mathrm{HF}$ or $\mathrm{PAH}$, excess ventilation often occurs with a rapid shallow breathing pattern, to compensate for the exertional pathophysiological condition, which has been postulated to be due to (1) earlier onset of exertional lactic acidosis, (2) increased chemoreflex activity, (3) a restrictive lung mechanism, (4) reduction in alveolar-capillary gas diffusion, and (5) increased sympathetic activity (Sue, 2011). In humans, regardless of the underlying disease, $\mathrm{pH}$-related homeostasis is commonly an important metabolic determinant of ventilatory control during exercise (Miki et al., 2009, 2012, 2013; Wasserman et al., 2014; 


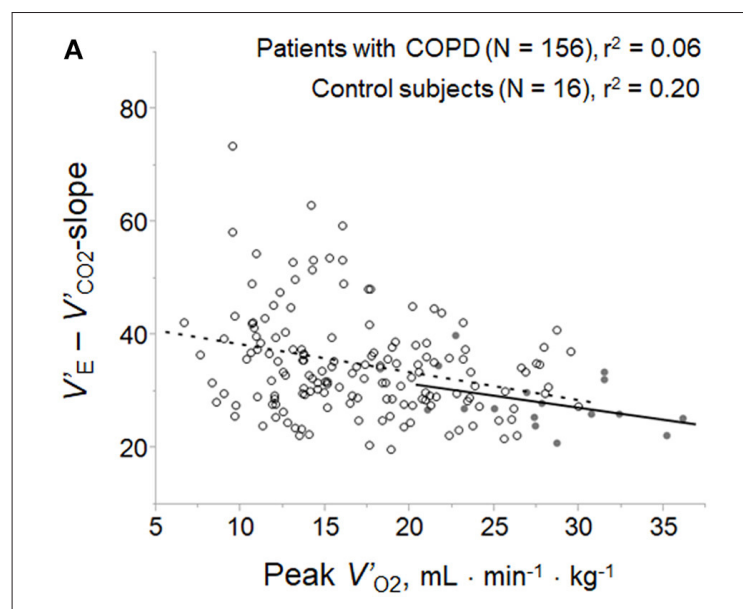

C

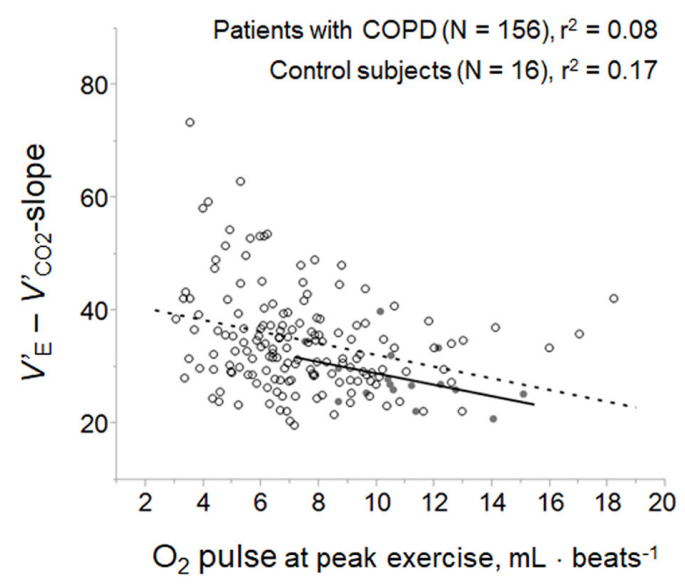

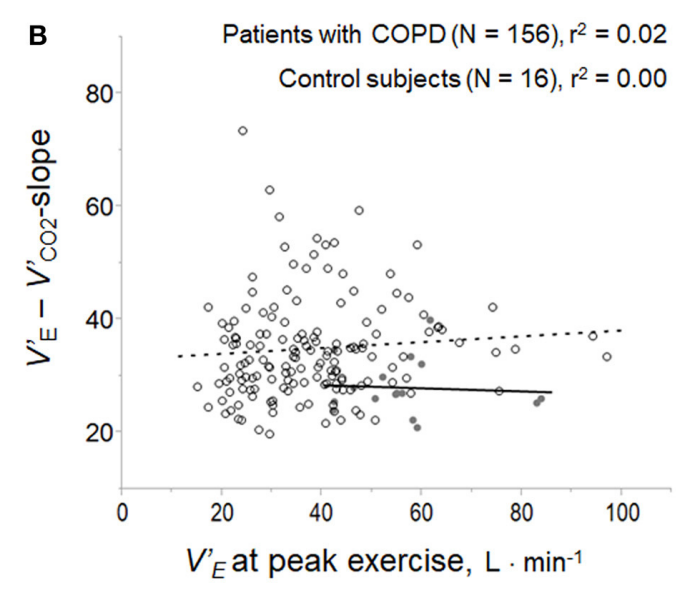

COPD, $\bigcirc \cdots \cdots . .$.

Control,

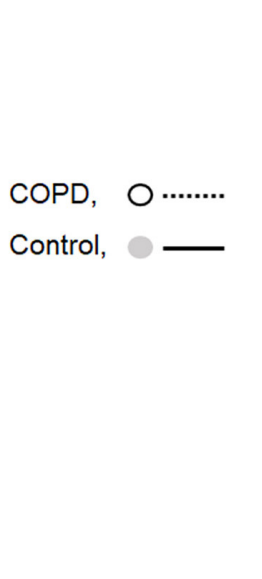

D

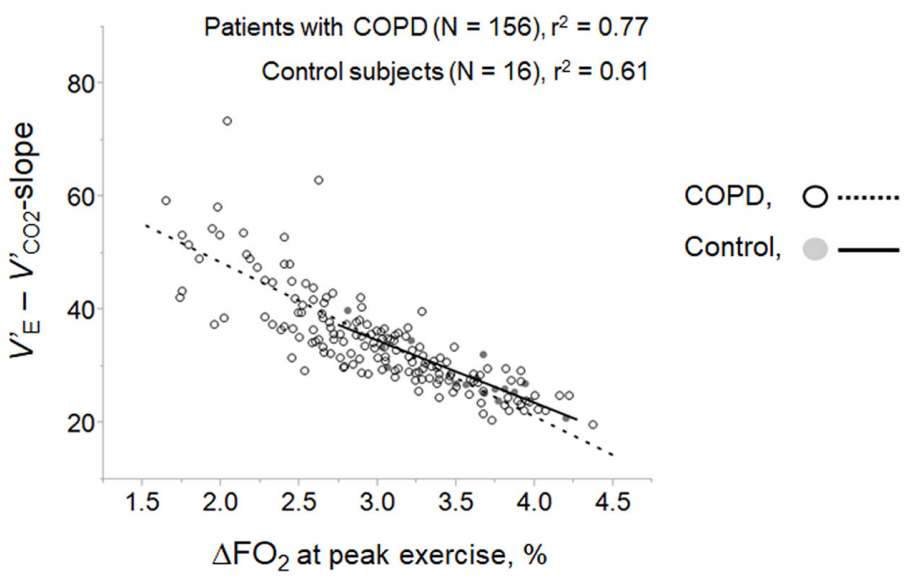

FIGURE 2 | Correlations of ventilatory inefficiency with (A) peak $V^{\prime} \mathrm{O}_{2}$, (B) $V^{\prime} \mathrm{E}$ at peak exercise, (C) $\mathrm{O}_{2}$ pulse at peak exercise, and (D) $\Delta \mathrm{FO} 2$ at peak exercise. $\Delta \mathrm{FO} \mathrm{O}_{2}$, difference between inspired and expired oxygen concentration; $\mathrm{O}_{2}$ pulse, $V^{\prime}{ }_{\mathrm{O} 2}$ /heart rate; $V^{\prime}{ }_{\mathrm{E}}$, minute ventilation; $V^{\prime}{ }_{\mathrm{O} 2}$, oxygen uptake.

Miki, 2021). Näveri et al. reported that the main limiting factor of exercise performance in both healthy subjects and $\mathrm{HF}$ patients is the development of lactic acidosis, although it occurs at different levels of exercise (Näveri et al., 1997). In the present study, in control subjects, exertional lactic acidosis occurred at peak exercise, followed by an adequate compensatory ventilatory response with tachypnea, which led to the low $V_{\mathrm{E}^{-}}^{\prime}$ $V^{\prime}{ }_{\mathrm{CO}}$-slope and $V_{\mathrm{E}}{ }^{\prime} / V^{\prime} \mathrm{CO}_{2}$-nadir. In $\mathrm{COPD}$, in contrast to $\mathrm{PAH}$ and $\mathrm{HF}$, there is limited evidence of a relationship between ventilatory inefficiency and the ventilatory response to exercise. Neder et al. reported that the $V_{\mathrm{E}}^{\prime}-V^{\prime} \mathrm{CO}_{2}$-slope decreases with the increasing severity of COPD (Neder et al., 2015). In the present study as well, increasing levels of severity of COPD were not associated with a high degree of ventilatory inefficiency, and the slow and shallow breathing with a prolonged expiratory pattern led to exertional respiratory acidosis due to wasted ventilation (Table 2). Further, in the present study, in contrast to cardiovascular disease (Sue, 2011; Weatherald and Laveneziana,
2018), the ventilatory inefficiency correlated well with the gas exchange parameters related to exertional respiratory acidosis rather than with exertional lactic acidosis levels (Table 5). This could be explained by the previously reported result that the resultant hypercapnia (O'Donnell et al., 2002) at which carbon dioxide $\left(\mathrm{CO}_{2}\right)$ is dominantly increased due to dynamic lung hyperinflation, led to a reduced $V_{\mathrm{E}}^{\prime}$ for a given $V^{\prime} \mathrm{CO}$, that is, low ventilatory inefficiency, in COPD. Interestingly, however, the ventilatory inefficiency related to $\mathrm{CO}_{2}$ clearance most strongly correlated with peak exercise $\Delta \mathrm{FO}_{2}$, which is considered to represent the sum of body's $\mathrm{O}_{2}$ extraction among $\mathrm{O}_{2}$ delivery, $\mathrm{O}_{2}$ reservoirs, and $\mathrm{O}_{2}$ consumption from the body's reservoirs in all subjects in the present study (Figures 2A-D and Table 5). In addition, there was no significant difference in the change in $\Delta \mathrm{FO}_{2}$ from rest to peak exercise or the change in $V_{\mathrm{E}}^{\prime} / V^{\prime}{ }_{\mathrm{CO} 2}$ from the value at rest to the nadir among all subjects in the present study (Table 2). Although little is so far known about the relationship between $\Delta \mathrm{FO}_{2}$ and the ventilatory response 
TABLE 5 | Correlation of the parameters related to $V^{\prime}{ }^{\prime}-V^{\prime}{ }^{\prime}{ }^{2}$-slope or $V^{\prime} \mathrm{E} / V^{\prime} \mathrm{CO}_{2}$-nadir in all control subjects and COPD patients $(n=172)$.

\begin{tabular}{|c|c|c|c|c|}
\hline & \multicolumn{2}{|c|}{$V_{\mathrm{E}}^{\prime}-V^{\prime} \mathrm{CO}_{2}$-slope } & \multicolumn{2}{|c|}{$V^{\prime}{ }_{E} / V^{\prime} \mathrm{CO}_{2}$-nadir } \\
\hline & $r$ & $p$-value & $r$ & $p$-value \\
\hline \multicolumn{5}{|c|}{ Peak incremental exercise parameters } \\
\hline Dyspnea, Borg scale & 0.0934 & 0.2245 & 0.0520 & 0.4995 \\
\hline$V_{\mathrm{O} 2}^{\prime}, \mathrm{mL} \cdot \mathrm{min}^{-1} \cdot \mathrm{kg}$ & -0.3219 & $<0.0001$ & -0.5493 & $<0.0001$ \\
\hline$V_{E}^{\prime}, L \cdot \min ^{-1}$ & 0.0479 & 0.5325 & 0.2178 & 0.0041 \\
\hline$V_{T}, m L$ & -0.0997 & 0.1932 & -0.3467 & $<0.0001$ \\
\hline$f_{\mathrm{R}}$, breaths $\cdot \mathrm{min}^{-1}$ & 0.2122 & 0.0052 & 0.1210 & 0.1137 \\
\hline Ti/Ttot & -0.0384 & 0.6172 & -0.2535 & 0.0008 \\
\hline$V_{\mathrm{E}}^{\prime} / V_{\mathrm{O} 2}^{\prime}$ & 0.8573 & $<0.0001$ & 0.8446 & $<0.0001$ \\
\hline$V_{D} / V_{T}$ & 0.3094 & $<0.0001$ & 0.5769 & $<0.0001$ \\
\hline $\mathrm{HR}$, beats $\cdot \mathrm{min}^{-1}$ & -0.2201 & 0.0037 & -0.3545 & $<0.0001$ \\
\hline $\mathrm{O}_{2}$ pulse, $\mathrm{mL} \cdot$ beats $^{-1}$ & -0.3331 & $<0.0001$ & -0.5615 & $<0.0001$ \\
\hline$\Delta \mathrm{FO}_{2}, \%$ & -0.8835 & $<0.0001$ & -0.9093 & $<0.0001$ \\
\hline $\mathrm{pH}$ & 0.4835 & $<0.0001$ & 0.4441 & $<0.0001$ \\
\hline $\mathrm{PaO}_{2}, \mathrm{mmHg}$ & -0.0686 & 0.3711 & -0.1776 & 0.0198 \\
\hline $\mathrm{PaCO}_{2}, \mathrm{mmHg}$ & -0.5250 & $<0.0001$ & -0.3357 & $<0.0001$ \\
\hline $\mathrm{PetCO}_{2}, \mathrm{mmHg}$ & -0.8542 & $<0.0001$ & -0.7819 & $<0.0001$ \\
\hline $\mathrm{HCO}_{3}^{-}, \mathrm{mEq} \cdot \mathrm{L}^{-1}$ & -0.3366 & $<0.0001$ & -0.1459 & 0.0561 \\
\hline Plasma lactate, $\mathrm{mg} \cdot \mathrm{dL}^{-1}$ & -0.1959 & 0.0105 & -0.3855 & $<0.0001$ \\
\hline \multicolumn{5}{|l|}{ Pulmonary function } \\
\hline $\mathrm{FEV}_{1}, \mathrm{~L}$ & 0.0419 & 0.5849 & -0.1993 & 0.0088 \\
\hline FVC, L & 0.1461 & 0.0559 & -0.0564 & 0.4628 \\
\hline IC, L & -0.0214 & 0.7809 & -0.1859 & 0.0146 \\
\hline \multicolumn{5}{|l|}{ Others } \\
\hline Age, y & 0.1498 & 0.0498 & 0.1988 & 0.0090 \\
\hline $\mathrm{BMI}, \mathrm{kg} \cdot \mathrm{m}^{-2}$ & -0.2906 & 0.0001 & -0.3746 & $<0.0001$ \\
\hline \multicolumn{5}{|c|}{ 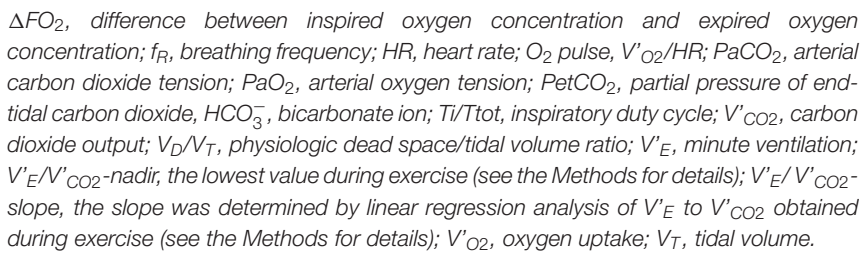 } \\
\hline
\end{tabular}

to exercise, ventilatory efficiency might depend on optimal $\mathrm{O}_{2}$ extraction and $\mathrm{CO}_{2}$ clearance in the cardiopulmonary-muscle crosstalk during exercise.

$\mathrm{O}_{2}$ extraction evaluated by $\Delta \mathrm{FO}_{2}$, which can be directly determined by non-invasive CPETs, correlated strongly with ventilatory inefficiency, and might contribute to compensating for the reduced exercise tolerance. The HR response to exercise in the present study showed that (1) HR at peak exercise and the quotient obtained by dividing the value by the predicted maximum $\mathrm{HR}$ decreased progressively, although the $\mathrm{HR}-V^{\prime} \mathrm{CO}^{-}$ slope increased from control to GOLD stage IV COPD (Table 2), that is, HR increased steeply during exercise because cardiac function decreased as the stage of COPD advanced, although the level of dysfunction was small; and (2) the peak exercise $\Delta \mathrm{FO}_{2}$ correlated with $\mathrm{O}_{2}$-pulse $(\mathrm{r}=0.4543)$, although it did not correlate well with the $\mathrm{HR}-V^{\prime} \mathrm{CO}_{2}$-slope, i.e., the degree of cardiac
TABLE 6 | Correlations of the parameters related to the difference between inspired oxygen concentration and expired oxygen concentration $\left(\Delta \mathrm{FO}_{2}\right)$ in all the study subjects and COPD patients.

\begin{tabular}{|c|c|c|c|c|}
\hline & \multicolumn{2}{|c|}{$\begin{array}{l}\text { All subjects } \\
\qquad(n=172)\end{array}$} & \multicolumn{2}{|c|}{$\begin{array}{l}\text { COPD patients } \\
\qquad(n=156)\end{array}$} \\
\hline & $r$ & $p$-value & $r$ & $p$-value \\
\hline \multicolumn{5}{|l|}{ Peak incremental exercise } \\
\hline Dyspnea, Borg scale & -0.0695 & 0.3677 & -0.0611 & 0.4504 \\
\hline$V_{\mathrm{O} 2}^{\prime}, \mathrm{mL} \cdot \mathrm{min}^{-1} \cdot \mathrm{kg}$ & 0.3728 & $<0.0001$ & 0.2951 & 0.0002 \\
\hline$V_{E}^{\prime}, L \cdot \min ^{-1}$ & 0.0058 & 0.9397 & -0.0991 & 0.2182 \\
\hline$V_{\mathrm{T}}, \mathrm{mL}$ & 0.1665 & 0.0295 & 0.0974 & 0.2262 \\
\hline$f_{\mathrm{R}}$, breaths $\cdot \mathrm{min}^{-1}$ & -0.2179 & 0.0042 & -0.2748 & 0.0005 \\
\hline $\mathrm{Ti} / \mathrm{Ttot}$ & 0.0795 & 0.3013 & -0.0538 & 0.5046 \\
\hline$V_{\mathrm{E}}^{\prime} / V^{\prime}{ }_{\mathrm{CO} 2}$ & -0.9223 & $<0.0001$ & -0.9189 & $<0.0001$ \\
\hline$V_{\mathrm{D}} / V_{\mathrm{T}}$ & -0.3655 & $<0.0001$ & -0.3077 & $<0.0001$ \\
\hline $\mathrm{pH}$ & -0.4062 & $<0.0001$ & -0.4202 & $<0.0001$ \\
\hline $\mathrm{PaO}_{2}, \mathrm{mmHg}$ & 0.0292 & 0.7043 & -0.0777 & 0.3352 \\
\hline $\mathrm{PaCO}_{2}, \mathrm{mmHg}$ & 0.4911 & $<0.0001$ & 0.5681 & $<0.0001$ \\
\hline $\mathrm{PetCO}_{2}, \mathrm{mmHg}$ & 0.8514 & $<0.0001$ & 0.8540 & $<0.0001$ \\
\hline $\mathrm{HCO}_{3}^{-}, \mathrm{mEq} \cdot \mathrm{L}^{-1}$ & 0.3283 & $<0.0001$ & 0.4078 & $<0.0001$ \\
\hline Plasma lactate, $\mathrm{mg} \cdot \mathrm{dL}^{-1}$ & 0.1406 & 0.0682 & 0.0817 & 0.3140 \\
\hline $\mathrm{HR}$, beats $\cdot \min ^{-1}$ & 0.1952 & 0.0105 & 0.1260 & 0.1172 \\
\hline $\mathrm{O}_{2}$ pulse, $\mathrm{mL} \cdot$ beats $^{-1}$ & 0.4543 & $<0.0001$ & 0.4050 & $<0.0001$ \\
\hline \multicolumn{5}{|l|}{ During incremental exercise } \\
\hline HR-V'cO2-slope, beats $\cdot L^{-1}$ & -0.1913 & 0.0122 & -0.1400 & 0.0812 \\
\hline \multicolumn{5}{|l|}{ Pulmonary function } \\
\hline $\mathrm{FEV}_{1}, \mathrm{~L}$ & 0.0275 & 0.7213 & -0.1147 & 0.1538 \\
\hline FVC, L & -0.0490 & 0.5242 & -0.1316 & 0.1014 \\
\hline $\mathrm{IC}, \mathrm{L}$ & 0.0772 & 0.3156 & 0.0195 & 0.8094 \\
\hline \multicolumn{5}{|l|}{ Others } \\
\hline Age, years old & -0.1408 & 0.0662 & -0.1243 & 0.1221 \\
\hline $\mathrm{BMI}, \mathrm{Kg} \cdot \mathrm{m}^{-2}$ & 0.3871 & $<0.0001$ & 0.4178 & $<0.0001$ \\
\hline
\end{tabular}

$\Delta \mathrm{FO}_{2}$, difference between inspired oxygen concentration and expired oxygen concentration; $f_{R}$, breathing frequency; $\mathrm{HR}$, heart rate; $\mathrm{O}_{2}$ pulse, $V^{\prime} \mathrm{O}_{2} / \mathrm{HR}$; $\mathrm{PaCO}_{2}$, arterial carbon dioxide tension; $\mathrm{PaO}_{2}$, arterial oxygen tension; $P$ etCO $\mathrm{C}_{2}$, partial pressure of endtidal carbon dioxide, $\mathrm{HCO}_{3}^{-}$, bicarbonate ion; $\mathrm{HR}-V^{\prime} \mathrm{CO}_{2}$-slope, the slope was determined by using linear regression analysis of HR to $V^{\prime} \mathrm{CO}_{2}$ obtained during the exercise (see the Methods for details); Ti/Ttot, inspiratory duty cycle; $V^{\prime} \mathrm{CO}_{2}$, carbon dioxide output; $V_{D} / V_{T}$, physiologic dead space/tidal volume ratio; $V_{E}^{\prime}$, minute ventilation; $V^{\prime}{ }^{2}$, oxygen uptake; $V_{T}$, tidal volume.

dysfunction in all subjects (Table 6). Given that the $\mathrm{O}_{2}$ pulse is equal to the product of stroke volume and the arteriovenous $\mathrm{O}_{2}$ difference, which depends on total $\mathrm{O}_{2}$ extraction from the body, and that $\Delta \mathrm{FO}_{2}$ did not correlate well with the $\mathrm{HR}-V^{\prime} \mathrm{CO}_{2}-$ slope or $V_{\mathrm{E}}^{\prime}$ at peak exercise in the present study, peak exercise $\Delta \mathrm{FO}_{2}$ might contribute to $\mathrm{O}_{2}$ extraction in the cardiopulmonary and peripheral muscle crosstalk rather than cardiac function or mechanical ventilation during exercise. In support of this, in the present study, a positive correlation between peak exercise $\Delta \mathrm{FO}_{2}$ and BMI was confirmed (Table 6), although the relationship between $\Delta \mathrm{FO}_{2}$ and muscle $\mathrm{O}_{2}$ extraction was not directly investigated in the present study. Interestingly, the $\Delta \mathrm{FO}_{2}-V^{\prime}$ $\mathrm{CO} 2$-slope and the ratio of $\Delta \mathrm{FO}_{2}$ to $V_{\mathrm{E}}^{\prime}$ at peak exercise increased progressively as the stage of COPD advanced, although, 
unexpectedly, there was no significant intrastage difference in peak exercise $\Delta \mathrm{FO}_{2}$ in the present study (Table 2 ). In addition, the $\Delta \mathrm{FO}_{2}-V^{\prime} \mathrm{CO}_{2}$-slope was positively correlated with the $\mathrm{PaCO}_{2}-V^{\prime} \mathrm{CO}_{2}$-slope, that is, the degree of exertional respiratory acidosis (Table 4). These findings suggest that (1) patients with enough exercise tolerance did not necessarily need a high peak exercise $\Delta \mathrm{FO}_{2}$, because such patients could depend on much of their ventilatory capability to increase oxygen uptake (Table 3 ) and (2) maintaining cardiopulmonary and peripheral muscle crosstalk is helpful to compensate for $\mathrm{O}_{2}$ demand, especially in patients with advanced COPD who predominantly have ventilatory impairments with exertional respiratory acidosis, because of the relatively high dependence of their reduced peak $V^{\prime}{ }^{\prime} 2$ on peak exercise $\Delta \mathrm{FO}_{2}$ compared with its dependence on $V^{\prime}$ E in GOLD IV cases (Table 3). This compensatory or protective mechanism of $\Delta \mathrm{FO}_{2}$ was not clearly proven in the present study. However, increasing the $\Delta \mathrm{FO}_{2}$ level or the slope of $\Delta \mathrm{FO}_{2}$ during exercise might be a therapeutic target for improving ventilatory inefficiency or exercise tolerance in COPD patients, this target being independent of the target of improving ventilatory capability (Figures 2B,D). Therefore, focusing on the two factors related to $V^{\prime}$ O2, that is, $\Delta \mathrm{FO}_{2}$ and $V^{\prime}$ E may be useful for developing therapeutic strategies to increase exercise tolerance. This is likely supported by the therapeutic variation pattern based on the two factors related to $V^{\prime} \mathrm{O} 2$, that is, the arteriovenous $\mathrm{O}_{2}$ difference and cardiac output that Cattadori et al. reported graphically to evaluate the effect of exercise training in HF patients (Cattadori et al., 2011). In addition, increased exertional muscle acidosis was associated with the down-regulation of skeletal muscle oxidative enzyme activity (Kutsuzawa et al., 1992). Recent evidence by Bruce et al. suggests that abnormal peripheral muscle metaboreflexes are responsible for the excess ventilation in COPD patients (Bruce et al., 2016). In cardiopulmonary diseases, identification of not only cardiopulmonary responses but also the peripheral muscle condition is commonly useful to facilitate personalized care based on each individual's dysfunction (Shelton et al., 2010; Vogiatzis and Zakynthinos, 2013; Maekura et al., 2015; Miki, 2021). In advanced COPD, measurement of $\Delta \mathrm{FO}_{2}$ might be informative for identifying ventilatory inefficiency, and might be a compensatory component for $\mathrm{O}_{2}$ availability in the body's cardiopulmonary and peripheral muscle crosstalk.

This study has some limitations. First, selection bias due to this being a single-center study, lack of data on pulmonary hypertension, and the low percentage of COPD patients regularly taking long-acting $\beta_{2}$-agonists and/or long-acting muscarinic antagonists might have affected the results. Second, to investigate the direct relationship between $\Delta \mathrm{FO}_{2}$ and muscle $\mathrm{O}_{2}$ extraction, the evaluation of peripheral exertional tissue oxygenation (Chuang et al., 2019), which was not performed in the present study might be more informative. Third, in cardiovascular disease, investigating of the relationship between ventilatory inefficiency and $\Delta \mathrm{FO}_{2}$ is necessary to confirm the increased appreciation of not only excess ventilation but also reduced $\mathrm{O}_{2}$ extraction as a marker of greater disease severity or worse outcomes. Given the report of Chuang et al. (2019), the conflicting evidence that excess ventilation is seen with the progression of cardiac disease, but not with the progression of COPD, might be related to the fact that exertional muscle oxygenation is more impaired in cardiac disease than in COPD as a pathophysiological mechanism of ventilatory inefficiency. Fourth, because many parameters in CPET are calculated using the basic parameters of gas flow, $\mathrm{O}_{2}$ concentration and $\mathrm{CO}_{2}$ concentration, multicollinearity due to variable selection should be evaluated. However, $\Delta \mathrm{FO}_{2}$ was directly determined as an actual value and was not used in the calculation of ventilatory inefficiency, with which it had the strongest correlation.

\section{CONCLUSION}

Ventilatory efficiency might depend on the exertional change in $\mathrm{O}_{2}$ extraction and $\mathrm{CO}_{2}$ clearance in the body. Measuring $\Delta \mathrm{FO}_{2}$ could be a key component for identifying ventilatory inefficiency related to $\mathrm{CO}_{2}$ clearance and $\mathrm{O}_{2}$ availability in the cardiopulmonary and peripheral muscle crosstalk of COPD. Individualized care to increase $\Delta \mathrm{FO}_{2}$ based on the specific dysfunction in COPD would help to improve both exercise tolerance and ventilatory inefficiency.

\section{DATA AVAILABILITY STATEMENT}

The raw data supporting the conclusions of this article will be made available by the authors, without undue reservation.

\section{ETHICS STATEMENT}

The studies involving human participants were reviewed and approved by the institutional review board of the National Hospital Organization Osaka Toneyama Medical Center. The patients/participants provided their written informed consent to participate in this study.

\section{AUTHOR CONTRIBUTIONS}

KM was responsible for the study conception and design. KM, $\mathrm{KT}, \mathrm{RM}, \mathrm{TM}, \mathrm{MM}, \mathrm{HH}, \mathrm{HK}, \mathrm{TKa}, \mathrm{TKu}$, and $\mathrm{HK}$ were responsible for data acquisition, analysis, interpretation, were responsible for drafting, and revising the article. Each author approved the submission of this manuscript for publication. All authors contributed substantially to this article.

\section{FUNDING}

This work was supported by a Grant-in-Aid for Clinical Research from the National Hospital Organization (number, not applicable). The funder had no role in the study design, data collection and analysis, or preparation of the manuscript.

\section{ACKNOWLEDGMENTS}

We would like to thank Mr. J. Ikeda, Ms. S. Sakaguchi, and S. Ito for help with the CPET measurements. 


\section{REFERENCES}

American Thoracic Society. (1995). Standardization of Spirometry, 1994 Update. Am. J. Respir. Crit. Care Med. 152, 1107-1136. doi: 10.1164/ajrccm.152.3.7663792

Bruce, R. M., Turner, A., and White, M. J. (2016). Ventilatory responses to muscle metaboreflex activation in chronic obstructive pulmonary disease. J. Physiol. 594, 6025-6035. doi: 10.1113/jp272329

Cattadori, G., Schmid, J. P., Brugger, N., Gondoni, E., Palermo, P., and Agostoni, P. (2011). Hemodynamic effects of exercise training in heart failure. J. Card Fail. 17, 916-922. doi: 10.1016/j.cardfail.2011.07.010

Chuang, M. L., Lin, I. F., and Hsieh, M. J. (2019). More impaired dynamic ventilatory muscle oxygenation in congestive heart failure than in chronic obstructive pulmonary disease. J. Clin. Med. 8:1641. doi: 10.3390/jcm8101641

Dubé, B. P., Agostoni, P., and Laveneziana, P. (2016). Exertional dyspnoea in chronic heart failure: the role of the lung and respiratory mechanical factors. Eur. Respir. Rev. 25, 317-332. doi: 10.1183/16000617.0048-2016

European Respiratory Society. (1997). Clinical exercise testing with reference to lung diseases: indications, standardization and interpretation strategies. ERS task force on standardization of clinical exercise testing. Eur. Respir. J. 10, 2662-2689. doi: 10.1183/09031936.97.10112662

Guazzi, M., Adams, V., Conraads, V., Halle, M., Mezzani, A., Vanhees, L., et al. (2012). EACPR/AHA scientific statement. Clinical recommendations for cardiopulmonary exercise testing data assessment in specific patient populations. Circulation 126, 2261-2274. doi: 10.1161/CIR.0b013e3182 $6 \mathrm{fb} 946$

Kutsuzawa, T., Shioya, S., Kurita, D., Haida, M., Ohta, Y., and Yamabayashi, H. (1992). 31P-NMR study of skeletal muscle metabolism in patients with chronic respiratory impairment. Am. Rev. Respir. Dis. 146, 1019-1024. doi: 10.1164/ajrccm/146.4.1019

Laviolette, L., and Laveneziana, P. (2018). "Exercise Testing in the prognostic evaluation of patients with lung and heart diseases," in Clinical Exercise Testing (ERS Monograph), eds. P. Palange, P. Laveneziana, J.A. Neder and S.A. Ward. (Sheffield: European Respiratory Society), 222-234.

Maekura, R., Hiraga, T., Miki, K., Kitada, S., Miki, M., Yoshimura, K., et al. (2015). Personalized pulmonary rehabilitation and occupational therapy based on cardiopulmonary exercise testing for patients with advanced chronic obstructive pulmonary disease. Int. J. Chron. Obstruct. Pulmon. Dis. 10, 1787-1800. doi: 10.2147/copd.S86455

Miki, K. (2021). Motor pathophysiology related to dyspnea in COPD evaluated by cardiopulmonary exercise testing. Diagnostics 11:364. doi: 10.3390/diagnostics11020364

Miki, K., Maekura, R., Hiraga, T., Hashimoto, H., Kitada, S., Miki, M., et al. (2009). Acidosis and raised norepinephrine levels are associated with exercise dyspnoea in idiopathic pulmonary fibrosis. Respirology 14, 1020-1026. doi: 10.1111/j.1440-1843.2009.01607.x

Miki, K., Maekura, R., Hiraga, T., Kitada, S., Miki, M., Yoshimura, K., et al. (2012). Effects of oxygen on exertional dyspnoea and exercise performance in patients with chronic obstructive pulmonary disease. Respirology 17, 149-154. doi: 10.1111/j.1440-1843.2011.02086.x

Miki, K., Maekura, R., Miki, M., Kitada, S., Yoshimura, K., Tateishi, Y., et al. (2013). Exertional acidotic responses in idiopathic pulmonary fibrosis: the mechanisms of exertional dyspnea. Respir. Physiol. Neurobiol. 185, 653-658. doi: 10.1016/j.resp.2012.11.008

Näveri, H. K., Leinonen, H., Kiilavuori, K., and Härkönen, M. (1997). Skeletal muscle lactate accumulation and creatine phosphate depletion during heavy exercise in congestive heart failure. Cause of limited exercise capacity? Eur. Heart J. 18, 1937-1945. doi: 10.1093/oxfordjournals.eurheartj. a015203

Nayor, M., Xanthakis, V., Tanguay, M., Blodgett, J. B., Shah, R. V., Schoenike, M., et al. (2020). Clinical and hemodynamic associations and prognostic implications of ventilatory efficiency in patients with preserved left ventricular systolic function. Circ. Heart Fail. 13:e006729. doi: 10.1161/circheartfailure.119.006729

Neder, J. A., Arbex, F. F., Alencar, M. C., O’Donnell, C. D., Cory, J., Webb, K. A., et al. (2015). Exercise ventilatory inefficiency in mild to end-stage COPD. Eur. Respir. J. 45, 377-387. doi: 10.1183/09031936.00135514

Neder, J. A., Berton, D. C., Arbex, F. F., Alencar, M. C., Rocha, A., Sperandio, P. A., et al. (2017). Physiological and clinical relevance of exercise ventilatory efficiency in COPD. Eur. Respir. J. 49(3). doi: 10.1183/13993003.02036-2016

O’Donnell, D. E., D’Arsigny, C., Fitzpatrick, M., and Webb, K. A. (2002). Exercise hypercapnia in advanced chronic obstructive pulmonary disease: the role of lung hyperinflation. Am. J. Respir. Crit. Care Med. 166, 663-668. doi: 10.1164/rccm.2201003

Radtke, T., Crook, S., Kaltsakas, G., Louvaris, Z., Berton, D., Urquhart, D. S., et al. (2019). ERS statement on standardisation of cardiopulmonary exercise testing in chronic lung diseases. Eur. Respir. Rev. 28:154. doi: 10.1183/16000617.0101-2018

Shelton, R. J., Ingle, L., Rigby, A. S., Witte, K. K., Cleland, J. G., and Clark, A. L. (2010). Cardiac output does not limit submaximal exercise capacity in patients with chronic heart failure. Eur. J. Heart Fail 12, 983-989. doi: 10.1093/eurjhf/hfq086

Sue, D. Y. (2011). Excess ventilation during exercise and prognosis in chronic heart failure. Am. J. Respir. Crit. Care Med. 183, 1302-1310. doi: 10.1164/rccm.201006-0965CI

Vogiatzis, I., and Zakynthinos, S. (2013). The physiological basis of rehabilitation in chronic heart and lung disease. J. Appl. Physiol. 115, 16-21. doi: 10.1152/japplphysiol.00195.2013

Wasserman, K., Cox, T. A., and Sietsema, K. E. (2014). Ventilatory regulation of arterial $\mathrm{H}(+)(\mathrm{pH})$ during exercise. Respir. Physiol. Neurobiol. 190, 142-148. doi: 10.1016/j.resp.2013.10.009

Wasserman, K., Hansen, J., Sue, D., Stringer, W., Sietsema, K., and Sun, X.-G. (2012). Principles of Exercise Testing and Interpretation: Including Pathophysiology and Clinical Applications. Philadelphia, PA: Lippincott Williams and Wilkins

Wasserman, K., Zhang, Y. Y., Gitt, A., Belardinelli, R., Koike, A., Lubarsky, L., et al. (1997). Lung function and exercise gas exchange in chronic heart failure. Circulation 96, 2221-2227. doi: 10.1161/01.cir.96.7.2221

Weatherald, J., and Laveneziana, P. (2018). "Patterns of cardiopulmonary response to exercise in pulmonary vascular diseases," in Clinical Exercise Testing (ERS Monograph), eds P. Palange, P. Laveneziana, J.A. Neder and S.A. Ward. (Sheffield: European Respiratory Society), 160-174.

Weatherald, J., Sattler, C., Garcia, G., and Laveneziana, P. (2018). Ventilatory response to exercise in cardiopulmonary disease: the role of chemosensitivity and dead space. Eur. Respir. J. 51:1700860. doi: 10.1183/13993003.00860-2017

Conflict of Interest: The authors declare that the research was conducted in the absence of any commercial or financial relationships that could be construed as a potential conflict of interest.

Publisher's Note: All claims expressed in this article are solely those of the authors and do not necessarily represent those of their affiliated organizations, or those of the publisher, the editors and the reviewers. Any product that may be evaluated in this article, or claim that may be made by its manufacturer, is not guaranteed or endorsed by the publisher.

Copyright (C) 2021 Miki, Tsujino, Maekura, Matsuki, Miki, Hashimoto, Kagawa, Kawasaki, Kuge and Kida. This is an open-access article distributed under the terms of the Creative Commons Attribution License (CC BY). The use, distribution or reproduction in other forums is permitted, provided the original author(s) and the copyright owner(s) are credited and that the original publication in this journal is cited, in accordance with accepted academic practice. No use, distribution or reproduction is permitted which does not comply with these terms. 